\title{
Urinary tract infection and antimicrobial resistance profile in patients attending Nemba District Hospital in Rwanda
}

\author{
Munyemana Jean Bosco', Bikorimana Jonas², Kantarama Evelyne', \\ Kabanyana Pauline ${ }^{3}$ \\ ${ }^{1}$ Department of Clinical Biology, School of Medicine and Pharmacy, College of Medicine and Health Sciences, \\ University of Rwanda, ${ }^{2}$ Department of Biomedical Laboratory Sciences, Faculty of Applied Fundamental Sciences, \\ INES-Ruhengeri, ${ }^{3}$ Department of Nursing, School of Nursing and Midwifery, College of Medicine and Health Sciences, \\ University of Rwanda, Kigali-Rwanda
}

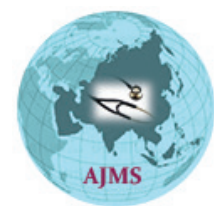

A B S TR A C T

Background: About 150 million people worldwide are diagnosed with urinary tract infection (UTI) every year and more than half of women get UTI at least once in their lifetimes. Overuse and misuse of antibiotics have contributed to the growing problem of resistance amongst uropathogenic bacteria making it hard to treat. Aims and Objectives: This study aimed to determine bacteria causing UTIs and their antibiotic resistance profile among patients attending Nemba District Hospital in Rwanda. Materials and Methods: A retrospective study design was used and 267 patient's data with positive urine culture were reviewed from microbiology laboratory logbooks. Results: In this study, UTI was more frequent in female $180(67.4 \%)$ than male $87(32.6 \%)$. The most frequently isolated bacteria to cause UTI were Escherichia coli (56.93\%) followed by Staphylococcus aureus $(28.46 \%)$, Proteus spp. (4.49\%), Klebsiella spp. (3.75\%), Morganella morganii (1.87\%), Coagulase Negative Staphylococcus (1.50\%), Neisseria gonorrhea (1.12\%), Enterobacter spp. $(1.12 \%)$ and Citrobacter spp. $(0.7 \%)$. There was a high antimicrobial resistance profile among bacterial isolates. The most commonly used antimicrobial agents including third-generation cephalosporin were resistant to bacterial isolates at a higher rate. Conclusion: These findings suggest continued antimicrobial resistance surveillance and special precautions should be taken for empirical treatment referring to local antimicrobial resistance.

Key words: Bacterial isolates; Antimicrobial resistance; Urinary tract infection
http://nepjol.info/index.php/AJMS DOI: 10.3126/ajms.v11i6.29921

E-ISSN: 2091-0576

P-ISSN: 2467-9100

Copyright (c) 2020 Asian Journal of Medical Sciences

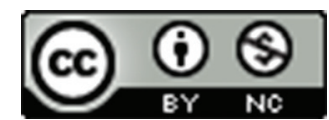

This work is licensed under a Creative Commons Attribution-NonCommercial 4.0 International License.

\section{INTRODUCTION}

Urinary tract infection (UTI) is among the common infections, nearly $10 \%$ of people experience it during their lifetime. UTI may be symptomatic or asymptomatic, community, or hospital acquired and can results in serious sequelae if left untreated. ${ }^{1}$ Although several different microorganisms can cause UTIs, bacteria are the major causative organisms and are responsible for more than $95 \%$ of UTI cases. ${ }^{2}$
Bacterial UTI is primarily caused by Gram-negative bacteria $^{3}$ evenGram-positive pathogens are also involved. ${ }^{4}$ More than $95 \%$ of uncomplicated UTIs are monobacteria infection ${ }^{5}$ and the most common pathogen responsible for it, are Eschericbia. Coli (E.coli)(75\%-95\%), followed by Klebsiella pneumoniae, Staphylococcus saprophyticus, Enterococcus faecalis, group B streptococci, and Protens mirabilis. E. coli can cause both uncomplicated and complicated UTIs while P. mirabilis, Pseudomonas aeruginosa and Enterococcus

Address for Correspondence:

Munyemana Jean Bosco, Department of Clinical Biology, School of Medicine and Pharmacy, College of Medicine and Health Sciences,

University of Rwanda. Phone:+250788226375. E-mail: munyebos1@gmail.com 
spp.predominantly cause complicated infections and are more commonly isolated in hospitals and long-term care facilities. $^{6}$

Millions of people worldwide are affected by urinary tract infections every year. ${ }^{7}$ There is a high increase in the prevalence of UTIs in Africa especially in sub-Saharan countries. ${ }^{7,8}$ In Rwanda, the previous study conducted in patients attending Butare University Teaching Hospital (BUTH) and Kigali University Teaching Hospital (KUTH) found that E. coli was the most common uropathogenic accounting $60.7 \%$ of UTI cases and frequently occurred in outpatients $(70.6 \%){ }^{9}$

The introduction of antibiotic therapy has played an important role in the management of UTIs. However, the major problem with current antimicrobial therapy is the rapid emergence of antibiotic resistance in both hospital and community acquired UTI cases. Antimicrobial sensitivity testing (AST) was found to be a solution and reliable guidance to antimicrobial therapy. Unfortunately, the low-income countries do not have a well-equipped and functioning microbiology laboratory to perform AST. In addition, turnaround time (TAT) of AST results is longer and cannot serve emergency cases. Thus, empirical treatment has become routine practice and the only solution especially in sub-Saharan countries. However, treatment failure associated to increased antimicrobial resistance is emerging. ${ }^{5,10}$

Initial appropriate empirical treatment requires a good knowledge of local and global epidemiological data; unfortunately, most of Sub-Saharan countries luck continued surveillance. Moreover, emergency and continuing antibiotic resistance phenomenon pause great challenge on empirical treatment, and pathogen spectrum resistance rates vary according to the geographical setting, suggesting continued and regular antimicrobial resistance monitoring to improve and revise empirical treatment guidelines. ${ }^{2,7} \mathrm{It}$ is in this regard, this study aimed to determine the most common bacteria causing UTIs and their antimicrobial resistance profile in patients attending Nemba District Hospital in Rwanda.

\section{MATERIAL AND METHODS}

\section{Study setting and design}

This study was conducted in the Northern Province of Rwanda, Gakenke District at Nemba District Hospital. It was a retrospective study design and data were collected from archived urine culture results logbook in the microbiology laboratory unit of Nemba District Hospital.

\section{Data collection}

Data were collected from $1^{\text {st }}$ July 2017 up to $30^{\text {th }}$ June 2019 and only positive urine culture cases were taken into consideration. Retrospectively, from microbiology logbooks, a total number of 267 cases suspected of having UTI with positive urine culture were included in the study.

\section{Data analysis}

Data were entered into Microsoft Excel and exported in SPSS version 22 for frequencies and percentages calculation. Data were presented in tables and figures.

\section{Ethical considerations}

The researcher handled all patients' data gathered in this study confidentially. Furthermore, laboratory anonymous coding was used to hide the identity of patients. Ethical clearance was obtained from the institutional review board of INES Ruhengeri and was presented to the administration of Nemba District Hospital for approval. Before starting data collection, an acceptance letter was given to the researcher form Nemba District Hospital administration.

\section{RESULTS}

\section{Characteristics of the study participants}

The current study has recruited 267 participants including 180(67.4\%) female and 87(32.6\%) male. The mean age of the participants was $43.7( \pm 20.3)$ years of age. The age groups of $\geq 15$ years were $23(8.6 \%), 15$-35 years were $78(29.2 \%)$, 36-50 years were $81(30.3 \%)$, while the participants $\geq 50$ years were $85(31.9 \%$ ) (Table 1 ).

\section{Frequency of bacterial isolates}

E. coli was the most isolate $152(57.0 \%)$ followed by S. aureus 76 (28.4\%), Proteus spp. 12 (4.4\%), Klebsiella spp. 10 (4.0\%), Morganella morganii 5 (2.0\%), Coagulase Negative

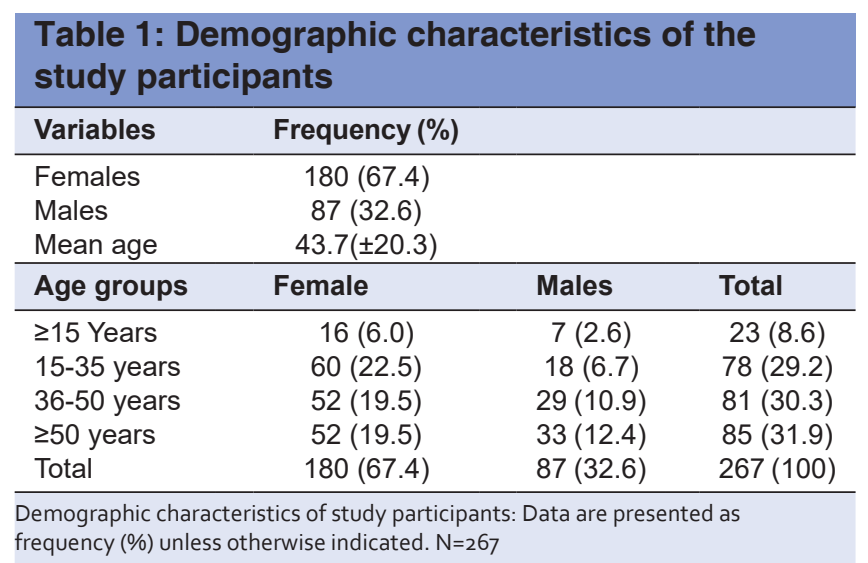


Staphylococcus (CNS) 4 (1.50\%), Neisseria gonorrboeae $3(1.1 \%)$, Enterobacter spp. $3(1.1 \%)$ and Citrobacter spp. $2(0.7 \%)$ (Figure 1). In addition, Gram Negative isolates accounted 187(70\%) while Gram Positive isolates were 80(30\%) (Figure 2).

Antimicrobial resistance profile of bacterial isolates The main Gram negative isolates were Escherichia coli and Klebsiella spp and exhibited antimicrobial resistance as following: E.coli was resistant to Gentamicin 28.3\%, Ciproflaxacin13,1\%, Norflaxacin31.6\%, Ampicillin 79.6\%, Oxacillin $(80.9 \%)$, Tetracycline(50\%), Cefotaxime(20.4), Doxycycline(65.1\%), Erythromycin(83.6\%), Naladixic Acid(31.6). Klebsiella spp was resistant to Gentamicin (40\%), Ciproflaxacin $(20 \%)$, Norflaxacin (30\%), Ampicillin (90\%), Oxacillin (80\%), Tetracycline (40\%), Cefotaxime (40\%), Doxycycline (70\%), Erythromycin (100\%), Naladixic Acid (10\%). The main Gram Positive isolate were $S$. aureus and were resistant to: Gentamicin (26.3\%), Ciproflaxacin (19.7\%), Norflaxacin $(32.9 \%)$, Ampicillin (78.9\%), Oxacillin (64.5\%), Tetracycline (65.8\%), Cefotaxime (27.6\%), Doxycycline (59.3\%), Erythromycin (86.9\%), Naladixic Acid (30.3\%) (Table 2).

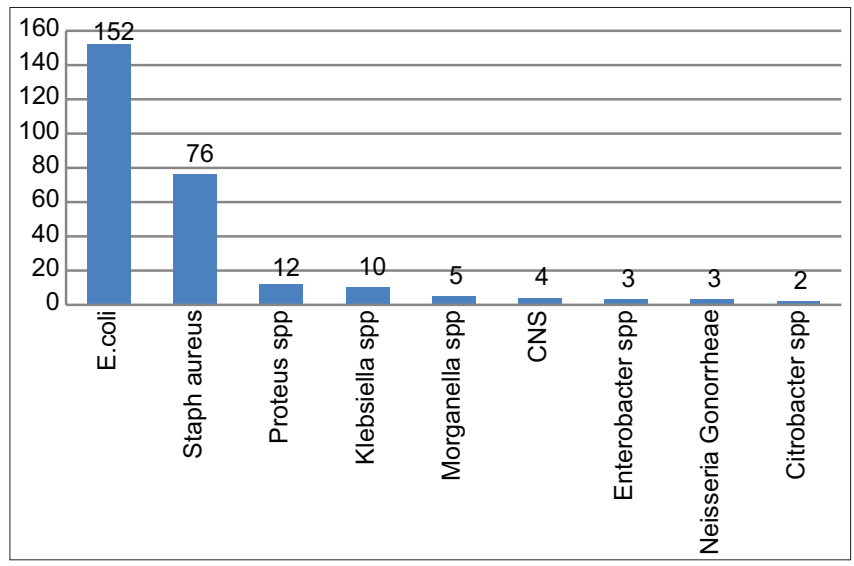

Figure 1: Frequency of bacteria species isolated from clinical specimen. Data are presented as frequency (\%) unless otherwise indicated. $\mathrm{N}=267$

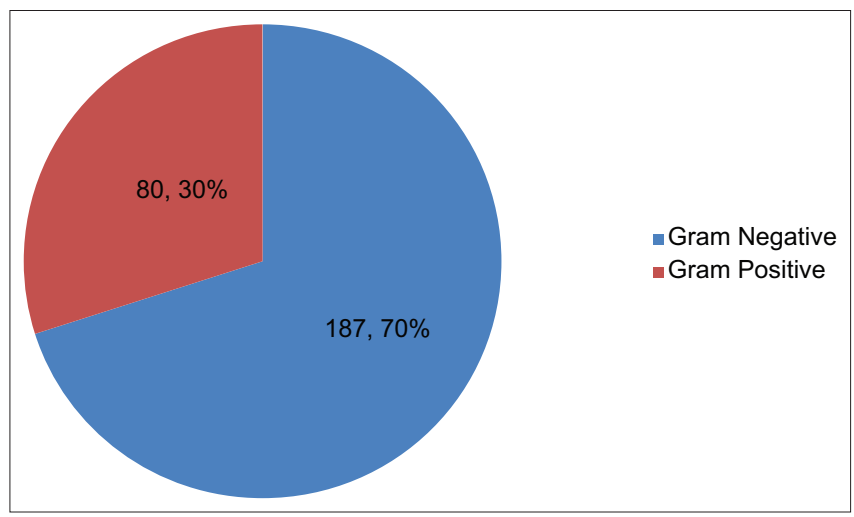

Figure 2: Frequency of Gram Negative and Gram Positive isolates. Data are presented as frequency (\%) unless otherwise indicated. $\mathrm{N}=267$

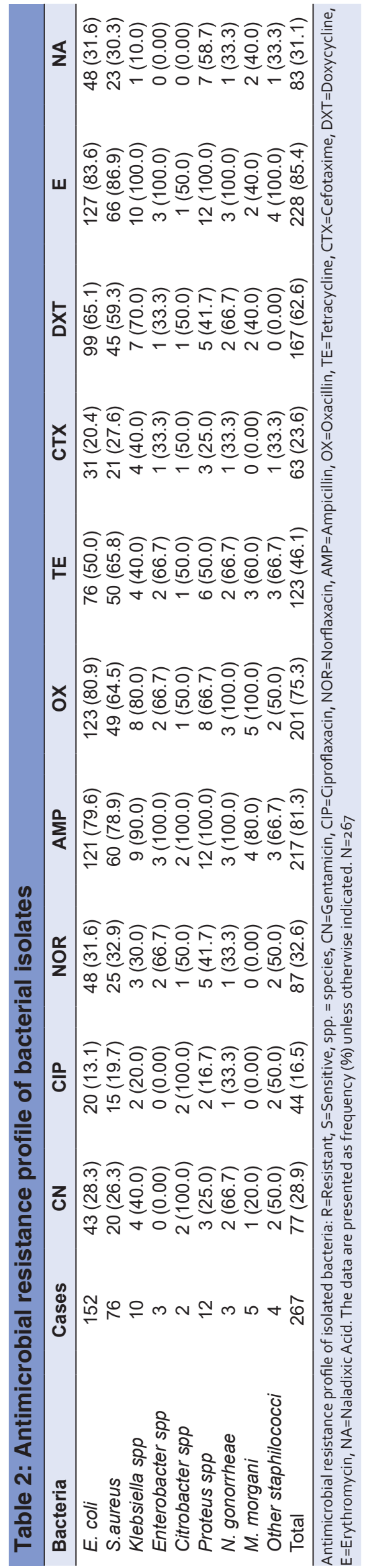

Asian Journal of Medical Sciences | Nov-Dec 2020 | Vol 11 | Issue 6 


\section{DISCUSSION}

The study included 267 participants with UTI. The infection was equally distributed in age groups above 15 years old and females were more affected than males. This is in agreement with the review of Mikolaj M et al where they highlight that UTIs are still a common clinical problem occurring more often in sexually active women, pregnancy, elderly, after catheterization of a urinary bladder and urological surgery as well as in the co-existence of diabetes or nephrolithiasis. ${ }^{11}$ The female predisposition may be associated with the anatomical structure of their genital urinary organ and gut normal flora, which can easily be transferred to the genital organ. Besides, fecal contamination could be associated with female UTI who have a shorter ureteral canal.

Also, the current study has found that Gram-negative bacteria were more involved in UTI than Gram-positive pathogens. Previously, Gram-negative bacteria especially Enterobacteriaceae family were reported to cause UTI. ${ }^{12}$ This could be due to the presence of a unique structure in Gram-negative bacteria, which facilitates attachment to the uroepithelial cell and their predominance in the gastrointestinal tract. Those unique characteristics prevent their elimination with urinary lavage and allow their multiplication, which may result in tissue invasion pyelonephritis. Another finding of the study is that, the main isolates were Gram-negative bacteria, mainly E.coli and Klebsiella spps. The finding is similar to that reported in the study conducted by Ntirenganya et al, where they found that E.coli was the main causative agent of UTI in Rwanda. ${ }^{13}$ Also, in our study, Gram-positive isolates were predominated by Staphylococcus aureus. Similar data were reported where E. coli was the main causative agent of UTI at a rate of $54.88 \%$, followed by $S$. aureus and Klebsiella spps. ${ }^{8}$ Similarly, in the Kabugo et al study, the E. coli was isolated at a $50 \%$ rate and followed by $S$. aureus with $15.4 \% .{ }^{14}$ All of those findings highlight that E.coli is the most UTI causative agent. This may be attributed to fecal contamination, as it is normal flora. ${ }^{11}$

Antimicrobial agents are the only option to manage bacterial infections; however, the emergency of their resistance is handicapping the prognosis. Epidemiological surveillance is only remaining guidance for empirical treatment. ${ }^{15,16} \mathrm{Thus}$, the current study has evaluated the antimicrobial resistance profile of isolated UTI causative agents. The finding of this study highlight increased resistance of commonly used drugs including third generation cephalosporin. The finding is in accordance with that of the previous study conducted by Ntirenganya $e t$ al, which reported an alarming rate of drug resistance among both gram-negative and gram-positive organisms ${ }^{13}$ in Rwanda. It is also consistent with the study of Kabugo et al conducted in Uganda where $50 \%$ of UTI isolated showed resistance to commonly used drugs $^{14}$. These results are also in the same line with the results from Ayelign et al study conducted in Turkey, where the resistance of isolated bacteria towards Ciprofloxacin was $80.88 \%$, Gentamicin was $79.41 \%$, and Tetracycline $58 \%$ and resistance of $72.06 \%$ to Ampicillin ${ }^{8}$. Taken all together, these findings clearly show how resistant strains are expanding at an alarming rate in the area. With this trend, an antibiotic, which was previously effective, might not be effective in the future.

\section{CONCLUSION}

The most UTI causative isolates were E. coli, S. aureus, Proteus spp., Klebsiella spp., Morganella morganii. UTI was more frequent in females than males and there was high antimicrobial resistance among bacterial isolates. The most commonly used antimicrobial agents including third generation cephalosporin were not susceptible to bacterial isolates at a higher rate. These findings suggest continued antimicrobial resistance surveillance and special precautions should be taken for empirical treatment.

\section{ACKNOWLEDGMENT}

We thank Nemba District Hospital administration to facilitate us to conduct our study at their health facility.

\section{REFERENCES}

1. Gupta K, Grigoryan L and Trautner B. Urinary Tract Infection. Annals of internal medicine. 2017;167(7) 3.

https://doi.org/10.7326/AITC201710030

2. Farajnia S, Alikhani MY, Ghotaslou R, Naghili B and Nakhlband A. Causative agents and antimicrobial susceptibilities of urinary tract infections in the northwest of Iran. International journal of infectious diseases : IJID : official publication of the International Society for Infectious Diseases. $2009 ; 13(2): 140-144$.

https://doi.org/10.1016/j.ijid.2008.04.014

3. Mazzariol A, Bazaj A and Cornaglia G. Multi-drug-resistant Gram-negative bacteria causing urinary tract infections: a review. Journal of chemotherapy. 2017;29(sup1):2-9. https://doi.org/10.1080/1120009X.2017.1380395

4. Kline $\mathrm{KA}$ and Lewis $\mathrm{AL}$. Gram-Positive Uropathogens, Polymicrobial Urinary Tract Infection, and the Emerging Microbiota of the Urinary Tract. Microbiology spectrum. 2016;4(2).

https://doi.org/10.1128/microbiolspec.UTI-0012-2012

5. Pietrucha-Dilanchian $P$ and Hooton TM. Diagnosis, Treatment, and Prevention of Urinary Tract Infection. Microbiology spectrum. $2016 ; 4(6)$.

https://doi.org/10.1128/microbiolspec.UTI-0021-2015

6. Foxman B. Urinary tract infection syndromes: occurrence, recurrence, bacteriology, risk factors, and disease burden. Infectious disease clinics of North America. 2014 ;28(1):1-13. 
https://doi.org/10.1016/j.idc.2013.09.003

7. Tandogdu Z and Wagenlehner FM. Global epidemiology of urinary tract infections. Current opinion in infectious diseases. $2016 ; 29(1): 73-79$.

https://doi.org/10.1097/QCO.0000000000000228

8. Ayelign B, Abebe B, Shibeshi A, Meshesha S, Shibabaw T, Addis $Z$, et al. Bacterial isolates and their antimicrobial susceptibility patterns among pediatric patients with urinary tract infections. Turkish journal of urology. 2018;44(1):62-69. https://doi.org/10.5152/tud.2017.33678

9. Muvunyi CM, Masaisa F, Bayingana C, Mutesa L, Musemakweri A, Muhirwa G, et al. Decreased susceptibility to commonly used antimicrobial agents in bacterial pathogens isolated from urinary tract infections in Rwanda: need for new antimicrobial guidelines. The American journal of tropical medicine and hygiene. 2011;84(6):923-928.

https://doi.org/10.4269/ajtmh.2011.11-0057

10. Yuksel S, Ozturk B, Kavaz A, Ozcakar B, Acar B, Guriz H, et al. Antibiotic resistance of urinary tract pathogens and evaluation of empirical treatment in Turkish children with urinary tract infections. 2006; 28(5):413-416.

https://doi.org/10.1016/j.jijantimicag.2006.08.009

11. Michno $M$ and Sydor $A$. Urinary tract infections in adults. Przeglad lekarski. 2016;73(7):504-508. 10.1111/j.1365-
2362.2008.020016

12. Byron JK. Urinary Tract Infection. The Veterinary clinics of North America Small animal practice. 2019;49(2):211-221. https://doi.org/10.1016/j.cvsm.2018.11.005

13. Ntirenganya $\mathrm{C}$, Manzi $\mathrm{O}$, Muvunyi $\mathrm{CM}$ and Ogbuagu $\mathrm{O}$. High prevalence of antimicrobial resistance among common bacterial isolates in a tertiary healthcare facility in Rwanda. The American journal of tropical medicine and hygiene. 2015;92(4): 865-870. https://doi.org/10.4269/ajtmh.14-0607

14. Kabugo D, Kizito S, Ashok DD, Graham KA, Nabimba R, Namunana S, et al. Factors associated with community-acquired urinary tract infections among adults attending assessment centre, Mulago Hospital Uganda. African health sciences. 2016;16(4):1131-1142. https://doi.org/10.4314/ahs.v16i4.31

15. Tadesse BT, Ashley EA, Ongarello S, Havumaki J, Wijegoonewardena M, Gonzalez IJ, et al. Antimicrobial resistance in Africa: a systematic review. BMC infectious diseases. 2017;17(1):616. https://doi.org/10.1186/s12879-017-2713-1

16. Essack SY, Desta AT, Abotsi RE and Agoba EE. Antimicrobial resistance in the WHO African region: current status and roadmap for action. Journal of public health. 2017;39(1):8-13. https://doi.org/10.1093/pubmed/fdw015

Author's contribution:

MJB and BJ- have designed the project and curried out the study. All authors have contributed in manuscript writing and correction.

Work attributed to:

Department of Clinical Biology, School of Medicine and Pharmacy, College of Medicine and Health Sciences, University of Rwanda.

Orcid ID:

Munyemana Jean Bosco- (10 https://orcid.org/0000-0003-2547-8070

Bikorimana Jonas- (iD https://orcid.org/0000-0002-7392-869X

Kantarama Evelyne- (10 https://orcid.org/0000-0002-5428-8914

Kabanyana Pauline- (D https://orcid.org/0000-0002-0253-268X

Source of Funding: None, Conflict of Interest: All authors declares no conflict of interest. 